\title{
One-Year Outcome After Percutaneous Coronary Intervention for Acute Coronary Syndrome \\ - An Analysis of 20,042 Patients From a Japanese Nationwide Registry -
}

\author{
Mitsuaki Sawano, MD, PhD; Shun Kohsaka, MD, PhD; Hideki Ishii, MD, PhD; \\ Yohei Numasawa, MD, PhD; Kyohei Yamaji, MD, PhD; Taku Inohara, MD, PhD; \\ Tetsuya Amano, MD, PhD; Yuji Ikari, MD, PhD; Masato Nakamura, MD, PhD
}

\begin{abstract}
Background: Acute coronary syndrome (ACS) hospital survivors experience a wide array of late adverse cardiac events, despite considerable advances in the quality of care. We investigated 30-day and 1-year outcomes of ACS hospital survivors using a Japanese nationwide cohort.
\end{abstract}

Methods and Results: We studied 20,042 ACS patients who underwent percutaneous coronary intervention (PCI) in 2017: 10,242 (51\%) with ST-elevation myocardial infarction (STEMI), 3,027 (15\%) with non-ST-elevation myocardial infarction (NSTEMI), and $6,773(34 \%)$ with unstable angina (UA). The mean ( \pm SD) age was $69.6 \pm 12.4$ years, $77 \%$ of the patients were men, and $20 \%$ had a previous history of PCI. The overall 30-day all-cause, cardiac, and non-cardiac mortality rates were $3.0 \%, 2.4 \%$, and $0.6 \%$, respectively. The overall 1-year incidence of all-cause, cardiac, and non-cardiac death was $7.1 \%, 4.2 \%$, and $2.8 \%$, respectively. Compared with UA patients, STEMI patients had a higher risk of all fatal events, non-fatal ischemic stroke, and acute heart failure, and NSTEMI patients had a higher risk of heart failure.

Conclusions: The results from our ACS hospital survivor $\mathrm{PCl}$ database suggest the need to improve care for the acute myocardial infarction population to lessen the burden of 30-day mortality due to ACS, heart failure, and sudden cardiac death, as well as 1-year ischemic stroke and heart failure events.

Key Words: Acute coronary syndrome; Acute myocardial infarction; Mortality; Outcome; Percutaneous coronary intervention

$\mathbf{R}$ evascularization via percutaneous coronary intervention (PCI) remains the cornerstone of care for patients presenting with acute coronary syndrome (ACS). ${ }^{1}$ The procedural technique has improved over time due to advances in pharmacotherapy, the application of coronary stents, and the establishment of patient care flows targeted at fulfilling performance and quality measures; ${ }^{\mathbf{2}, 3}$ however, patients presenting with ACS remain susceptible to a wide array of late adverse cardiac events, such as sudden cardiac death, stent thrombosis, recurrent ACS, stroke, and heart failure. ${ }^{\mathbf{4 5}}$ Large-scale observational studies have been performed to investigate the real-world outcomes of revascularized patients. ${ }^{\mathbf{6} 8}$ However, the lack

\section{Editorial p1768}

of comprehensively collected data extending beyond crosssectional studies, administrative databases, or clinical trials has hindered the process of identifying and delivering appropriate care for ACS patients in the contemporary era.

In Japan, approximately 260,000 PCIs are performed annually, mainly for the purposes of operator and institutional certification by the Cardiovascular Intervention and Therapeutics (CVIT), the leading academic organization for cardiovascular and endovascular interventions. ${ }^{9}$ The J-PCI OUTCOME project was developed in collaboration

Received February 5, 2021; revised manuscript received April 25, 2021; accepted May 10, 2021; J-STAGE Advance Publication released online June 24, 2021 Time for primary review: 48 days

Department of Cardiology, Keio University School of Medicine, Tokyo (M.S., S.K., T.I.); Department of Cardiology, Fujita Health University Bantane Hospital, Nagoya (H.I.); Department of Cardiology, Japanese Red Cross Ashikaga Hospital, Ashikaga (Y.N.); Department of Cardiology, Kokura Memorial Hospital, Kitakyushu (K.Y.); Department of Cardiology, Aichi-Medical University, Nagakute (T.A.); Department of Cardiovascular Medicine, Tokai University, Isehara (Y.I.); and Division of Cardiovascular Medicine, Toho University Ohashi Medical Center, Tokyo (M.N.), Japan

Mailing address: Mitsuaki Sawano, MD, PhD, Department of Cardiology, Keio University School of Medicine, 35 Shinanomachi, Shinjuku-ku, Tokyo 160-8582, Japan. E-mail: mitsuakisawano@gmail.com

All rights are reserved to the Japanese Circulation Society. For permissions, please e-mail: cj@j-circ.or.jp

ISSN-1346-9843 


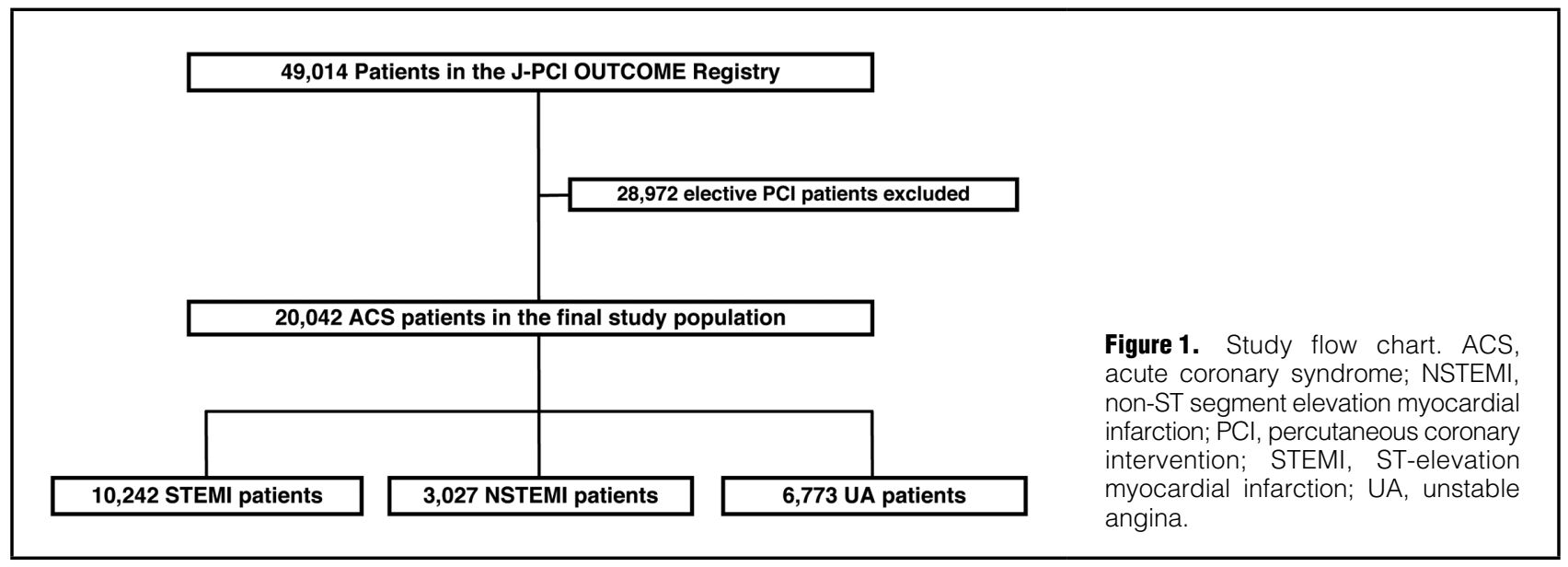

with the Japanese Circulation Society (JCS) and funded by the Japan Agency for Medical Research and Development to collect 1-year follow-up information for patients in the J-PCI Registry. This registry was the first of its kind to capture incident fatal and non-fatal events after initial PCI, including repeat revascularization at a nationwide scale, without linking to administrative databases for purpose of acquiring outcome data. The overall objective of establishing the registry was to reveal the diverse post-discharge status of care provided by PCI hospitals in Japan.

For this initial analysis, we used the J-PCI OUTCOME registry established in 2017, which extended the cross-sectional procedure-based J-PCI Registry to a nationwide longitudinal cohort that covered all 47 prefectures from 8 regions of Japan (Hokkaido, Tohoku, Kanto, Chubu, Kinki, Chugoku, Shikoku, and Kyushu/Okinawa) to report the 1-year outcomes of ACS hospital survivors and identify any potential problems and possible solutions to the current status of care as a foundation for quality improvement.

\section{Methods}

\section{The J-PCI and J-PCI OUTCOME Registries}

The J-PCI Registry is an ongoing prospective multicenter nationwide PCI registry led by the CVIT. ${ }^{10}$ The J-PCI Registry aims to provide national, regional, and institutional information on general aspects of patients, including background, clinical presentation, angiographic and procedural details, and in-hospital outcomes, as a foundation for further quality improvement. ${ }^{11-13}$ In addition, the J-PCI Registry has been incorporated into the National Clinical Data system, a nationwide prospective web-based registry linked to medical and surgical board certification, since 2013. ${ }^{14}$ Cardiac catheterization procedures are performed in both publicly and privately funded hospitals in Japan, and because registration in the J-PCI database is mandatory for both systems, data completeness is high. Based on annual reports from the insurance claims data, it is estimated that approximately $85 \%$ of all PCI procedures are registered in the J-PCI Registry.

Over 1,000 representative PCI centers from the 47 prefectures in Japan that fulfilled the selection criteria of exceeding an annual PCI case volume of 200 procedures $^{15}$ performed at each standardized performance site and were invited to participate in the J-PCI OUTCOME study in
December 2017. In all, 599 hospitals responded and 179 hospitals volunteered to participate and proceeded with the study protocol (Supplementary Appendix). The 172 institutions that registered over 10 patients during the study period were included in the final analysis to ensure data quality (Supplementary Figure).

Each center provided data on PCI patient outcomes. One-year mortality including all-cause death, cardiac death with information as to the mode of death, non-fatal events necessitating hospitalization for ACS, stroke, bleeding, acute heart failure, and repeat revascularization were registered for patients in the cohort. All 1-year outcome data were registered from August 2018 to March 2019 via a secure web-based electronic data capture system managed by the National Clinical Database with linkage to the J-PCI 2017 registry. Data were collected by data entry personnel either by reviewing health record charts or by contacting individual patients by telephone, and were reviewed by data managers responsible for each site. The final data were fixed in September 2019, and the analysis for the present study was performed from November 2019 to January 2020.

The study protocol was approved by a third-party central ethics committee at the Clinical Research Promotion Network Japan and local institutional review boards of each site. Patient consent was obtained through an opt-out format notification on websites and posters, and the procedures were conducted in accordance with the principles of the Declaration of Helsinki.

\section{Patient Information}

The participating hospitals included in the J-PCI OUTCOME cohort covered all 8 regions of Japan (Hokkaido, Tohoku, Kanto, Chubu, Kinki, Chugoku, Shikoku, and Kyushu/ Okinawa). For the present analysis, patients who underwent PCI from January 1, 2017 to December 31, 2017 were selected to form a patient-based cohort from a consecutively collected case-based 2017 J-PCI Registry. The date of the follow-up started from the date when the PCI preformed. All ACS patients that survived and were discharged after hospitalization were included in this study. Patient and PCI characteristics comparing the study population in the J-PCI OUTCOME cohort and 2017 J-PCI Registry are provided in Supplementary Table 1. The proportion of ACS and chronic kidney disease (CKD) patients and patients treated for proximal coronary lesions was 


\begin{tabular}{|c|c|c|c|c|}
\hline & $\begin{array}{c}\text { STEMI } \\
(n=10,242)\end{array}$ & $\begin{array}{l}\text { NSTEMI } \\
(\mathrm{n}=3,027)\end{array}$ & $\begin{array}{c}\text { UA } \\
(n=6,773)\end{array}$ & $P$ value \\
\hline Age (years) & $68.3 \pm 13.0$ & $70.0 \pm 12.5$ & $70.7 \pm 11.4$ & $<0.001$ \\
\hline \multicolumn{5}{|l|}{ Age group } \\
\hline$<65$ years & 3,539 (35) & $896(30)$ & $1,744(26)$ & \multirow{3}{*}{$<0.001$} \\
\hline 65 years $\leq$ age $<75$ years & $3,109(30)$ & $922(30)$ & 2,256 (33) & \\
\hline 75 years $\leq$ age $<80$ years & $3,594(35)$ & $1,209(40)$ & $2,773(41)$ & \\
\hline$\geq 80$ years & 2,265 (22) & $781(26)$ & $1,620(24)$ & $<0.001$ \\
\hline Male sex & $7,862(77)$ & $2,303(76)$ & $5,080(75)$ & $<0.001$ \\
\hline Previous PCI & $1,137(11)$ & 589 (19) & $2,260(33)$ & $<0.001$ \\
\hline Previous CABG & $104(1)$ & $104(3)$ & $316(5)$ & $<0.001$ \\
\hline Previous MI & $875(9)$ & $479(16)$ & $1,198(18)$ & $<0.001$ \\
\hline Previous HF & $461(5)$ & $348(11)$ & $789(12)$ & $<0.001$ \\
\hline Diabetes & 3,478 (34) & $1,161(38)$ & $2,856(42)$ & $<0.001$ \\
\hline Hypertension & $6,817(67)$ & $2,203(73)$ & $5,154(76)$ & $<0.001$ \\
\hline Dyslipidemia & $5,637(55)$ & $1,820(60)$ & $4,469(66)$ & $<0.001$ \\
\hline Smoking & $4,237(41)$ & $1,139(38)$ & 2,307 (34) & $<0.001$ \\
\hline CKD & $1,562(15)$ & $632(21)$ & $1,302(19)$ & $<0.001$ \\
\hline Hemodialysis & $230(2)$ & $150(5)$ & $468(7)$ & $<0.001$ \\
\hline COPD & $245(2)$ & $79(3)$ & $163(2)$ & $<0.001$ \\
\hline AAA/PAD & $301(3)$ & $177(6)$ & $454(7)$ & $<0.001$ \\
\hline Cardiogenic shock & $1,300(13)$ & $256(8)$ & $116(2)$ & $<0.001$ \\
\hline Cardiac arrest & $726(7)$ & $152(5)$ & $59(1)$ & $<0.001$ \\
\hline Any antiplatelet use prior to $\mathrm{PCl}$ & $8,298(81)$ & $2,612(86)$ & $6,091(90)$ & $<0.001$ \\
\hline Aspirin & $8,154(80)$ & $2,564(85)$ & $5,907(87)$ & $<0.001$ \\
\hline Clopidogrel & $1,129(11)$ & $601(20)$ & $1,958(29)$ & $<0.001$ \\
\hline Prasugrel & $6,410(63)$ & $1,747(58)$ & $3,458(51)$ & $<0.001$ \\
\hline Ticagrelor & $8(0)$ & $1(0)$ & $4(0)$ & $<0.001$ \\
\hline Other & $74(1)$ & $40(1)$ & $153(2)$ & $<0.001$ \\
\hline Any oral anticoagulant use & $377(4)$ & $170(6)$ & $397(6)$ & $<0.001$ \\
\hline Warfarin & $181(2)$ & $76(3)$ & $182(3)$ & $<0.001$ \\
\hline Dabigatran & $15(0)$ & $11(0)$ & $20(0)$ & $<0.001$ \\
\hline Rivaroxaban & $50(0)$ & $31(1)$ & $74(1)$ & $<0.001$ \\
\hline Apixaban & $53(1)$ & $32(1)$ & $64(1)$ & $<0.001$ \\
\hline Edoxaban & $36(0)$ & $19(1)$ & $46(1)$ & $<0.001$ \\
\hline Other & $62(1)$ & $12(0)$ & $18(0)$ & $<0.001$ \\
\hline Preprocedural Hb (g/dL) & $13.8 \pm 2.2$ & $13.3 \pm 2.2$ & $13.2 \pm 2.1$ & $<0.001$ \\
\hline Preprocedural Cr (mg/dL) & $0.9[0.7-1.1]$ & $0.9[0.7-1.1]$ & $0.9[0.7-1.1]$ & $<0.001$ \\
\hline Median (Q1-Q3) if STEMI & $70[53-90]$ & NA & NA & \\
\hline Door-to-balloon time $\leq 90 \mathrm{~min}$ & 6,806 & NA & NA & \\
\hline
\end{tabular}

Unless indicated otherwise, data are presented as the mean $\pm S D$, median [interquartile range], or $n(\%)$. AAA, abdominal aortic aneurysm; CABG, coronary artery bypass grafting; CKD, chronic kidney disease; COPD, chronic obstructive pulmonary disease; $\mathrm{Cr}$, creatinine; $\mathrm{Hb}$, hemoglobin; $\mathrm{HF}$, heart failure; MI, myocardial infarction; NSTEMI, non-ST segment elevation myocardial infarction; PAD, peripheral artery disease; $\mathrm{PCl}$, percutaneous coronary intervention; STEMI, ST-elevation myocardial infarction; UA, unstable angina.

higher in the OUTCOME cohort, whereas the proportion of patients with a previous history of PCI or myocardial infarction was lower than in the 2017 J-PCI Registry.

Data completeness of all collected variables was $>95 \%$, with the exception of door-to-balloon time, total fluoroscopy time, total procedure time, total anesthesia time, preprocedural hemoglobin level, and preprocedural creatinine level, which were newly added from January 2017 as optional entry variables.

\section{Definition of Key Variables}

ACS patients were selected as the main study population after excluding patients receiving PCI for elective indica- tions (Figure 1). Further, the ACS population was subdivided according to the clinical presentation of ST-elevation myocardial infarction (STEMI), non-ST-elevation myocardial infarction (NSTEMI), and unstable angina (UA). All ACS categories were diagnosed based on clinical presentation, electrocardiographic findings, and elevation of serum cardiac troponin or creatinine kinase concentrations.

Cardiogenic shock was defined as a sustained episode of systolic blood pressure $<80 \mathrm{mmHg}$ and/or a cardiac index of $<1.8 \mathrm{~L} \cdot \mathrm{min}^{-1} \cdot \mathrm{m}^{-2}$ (regardless of the measurement methods), despite the maximum treatment and determined to be secondary to cardiac dysfunction, and/or the need for parenteral inotropic or vasopressor agents or mechanical 


\begin{tabular}{|c|c|c|c|c|}
\hline & $\begin{array}{c}\text { STEMI } \\
(\mathrm{n}=10,242)\end{array}$ & $\begin{array}{c}\text { NSTEMI } \\
(\mathrm{n}=3,027)\end{array}$ & $\begin{array}{c}\text { UA } \\
(n=6,773)\end{array}$ & $P$ value \\
\hline \multicolumn{5}{|l|}{ Access site } \\
\hline Transfemoral & 3,798 (37) & $847(27)$ & $1,554(23)$ & $<0.001$ \\
\hline Transradial & $6,086(59)$ & $2,074(28)$ & $4,864(72)$ & \\
\hline Other & $358(3)$ & $106(69)$ & $355(5)$ & \\
\hline \multicolumn{5}{|l|}{ No. vessels treated during $\mathrm{PCl}$} \\
\hline One & $6,301(62)$ & $1,600(53)$ & $3,983(59)$ & $<0.001$ \\
\hline Two & $2,544(25)$ & $845(28)$ & $1,783(26)$ & 0.002 \\
\hline Three & $1,387(14)$ & $572(19)$ & 997 (15) & $<0.001$ \\
\hline LMT Iesion & $385(4)$ & $180(6)$ & $317(5)$ & $<0.001$ \\
\hline \multicolumn{5}{|l|}{ Treated coronary lesion location } \\
\hline Proximal lesion & $5,309(52)$ & $1,528(50)$ & $3,535(52)$ & 0.28 \\
\hline $\mathrm{PCl}$ to $\mathrm{RCA}$ & $4,148(40)$ & $865(29)$ & $2,190(32)$ & $<0.001$ \\
\hline $\mathrm{PCl}$ to $\mathrm{LCX}$ & $5,504(54)$ & $1,486(49)$ & $3,762(56)$ & $<0.001$ \\
\hline $\mathrm{PCl}$ to $\mathrm{LAD}$ & $1,481(14)$ & $1,101(36)$ & $1,544(23)$ & $<0.001$ \\
\hline $\mathrm{PCl}$ to graft lesion & $16(0.20)$ & $21(1)$ & $47(1)$ & $<0.001$ \\
\hline $\mathrm{PCl}$ to other lesion & $11(0.10)$ & $6(0.20)$ & $9(0.10)$ & $<0.001$ \\
\hline $\mathrm{PCl}$ to $\mathrm{LMT}$ & $441(4)$ & $242(8)$ & $440(6)$ & $<0.001$ \\
\hline \multicolumn{5}{|l|}{ Devices used during PCI } \\
\hline Drug-eluting stent & $8,835(86)$ & $2,532(84)$ & $5,797(86)$ & $<0.001$ \\
\hline Bare-metal stent & $139(1.40)$ & $30(1.00)$ & $63(0.90)$ & 0.03 \\
\hline POBA & $8,456(83)$ & $2,580(85)$ & $5,761(85)$ & $<0.001$ \\
\hline Drug-eluting balloon & $532(5)$ & $291(10)$ & $960(14)$ & $<0.001$ \\
\hline Thrombus aspiration & $5,809(57)$ & $771(25)$ & $500(7)$ & $<0.001$ \\
\hline Rotational atherectomy & $60(1)$ & $86(3)$ & $330(5)$ & $<0.001$ \\
\hline Direct cutting atherectomy & $2(0.02)$ & $0(0)$ & $13(0.19)$ & $<0.001$ \\
\hline Distal protection device & $1,014(10)$ & $124(4)$ & $308(5)$ & $<0.001$ \\
\hline Other devices & 309 (3) & $68(2)$ & $117(2)$ & $<0.001$ \\
\hline Unknown device & $4(0.04)$ & $2(0.07)$ & $2(0.03)$ & 0.7 \\
\hline \multicolumn{5}{|l|}{$\mathrm{PCl}$ success } \\
\hline Guidewire lesion penetration & $10,113(99)$ & $2,936(97)$ & $6,664(98)$ & $<0.001$ \\
\hline Fluoroscopy time (min) & $14[17-36]$ & $27[18-40]$ & $24[16-36]$ & $<0.001$ \\
\hline Procedure time (min) & 91 [69-123] & $95[70-130]$ & 90 [66-120] & $<0.001$ \\
\hline Periprocedural complication & $402(4)$ & $93(3)$ & $149(2)$ & $<0.001$ \\
\hline Cardiac tamponade & $22(0.20)$ & $1(0.00)$ & $5(0.10)$ & 0.01 \\
\hline Acute heart failure & $248(2)$ & $53(2)$ & $36(1)$ & $<0.001$ \\
\hline Acute stent thrombosis & $45(0.40)$ & $5(0.20)$ & $6(0.10)$ & $<0.001$ \\
\hline Access site bleeding & $24(0.20)$ & $9(0.30)$ & $8(0.10)$ & 0.12 \\
\hline Non-access site bleeding & $34(0.30)$ & $8(0.30)$ & $8(0.10)$ & 0.02 \\
\hline Emergency heart surgery & $12(0.10)$ & $4(0.10)$ & $7(0.10)$ & 0.9 \\
\hline
\end{tabular}

Unless indicated otherwise, data are given as the median [interquartile range] or $n(\%)$. LAD, left anterior descending; LCX, left circumflex; LMT, left main trunk; POBA, plain old balloon angioplasty; RCA, right coronary artery. Other abbreviations as in Table 1.

support, including an intra-aortic balloon pump and percutaneous mechanical circulatory device (e.g., venoarterial extracorporeal membrane oxygenation device) to maintain blood pressure and the cardiac index above the specified levels within the $24 \mathrm{~h}$ prior to PCI. Other definitions of variables included in the J-PCI Registry are available online (http://www.cvit.jp/registry/jpci_definition.pdf).

\section{Definitions of Clinical Outcomes}

Clinical endpoints were defined according to the 2017 Cardiovascular and Stroke Endpoint Definitions for Clinical Trials definitions. ${ }^{16}$ Definite stent thrombosis was registered for cardiovascular deaths and non-fatal ACS events according to the Academic Research Consortium-2 Consensus Document. ${ }^{17}$ A maximum of 3 events for non-fatal ACS, stroke, bleeding, and acute heart failure and 5 events for planned revascularization were registered in the database. Mode of death was divided into non-cardiac death, death due to unknown cause, cardiac death with subcategories of death due to ACS, sudden cardiac death, death due to heart failure, death due to stroke, death due to procedural complication, and death due to bleeding events. ACS patients were subdivided into those presenting with STEMI, NSTEMI, and UA with or without revascularization. Bleeding events were subdivided into access site bleeding, upper gastrointestinal bleeding, lower gastroin- 


\begin{tabular}{|c|c|c|c|c|}
\hline & $\begin{array}{c}\text { STEMI } \\
(\mathrm{n}=10,242)\end{array}$ & $\begin{array}{c}\text { NSTEMI } \\
(\mathrm{n}=3,027)\end{array}$ & $\begin{array}{c}\text { UA } \\
(n=6,773)\end{array}$ & $P$ value \\
\hline All-cause death ( 30 days) & $431(4)$ & $102(3)$ & $73(1)$ & $<0.001$ \\
\hline Cardiac death & $356(3)$ & $83(3)$ & $49(1)$ & 0.008 \\
\hline Cardiac death due to ACS & $270(3)$ & $60(2)$ & $19(0.3)$ & \multirow{6}{*}{$<0.001$} \\
\hline Cardiac death due to sudden cardiac arrest & $18(0.2)$ & $3(0.1)$ & $7(0.1)$ & \\
\hline Cardiac death due to AHF & $63(0.6)$ & $20(0.7)$ & $21(0.3)$ & \\
\hline Cardiac death due to stroke & $2(0.02)$ & $0(0)$ & $0(0)$ & \\
\hline Cardiac death due to procedural complications & $1(0.01)$ & $0(0)$ & $1(0.01)$ & \\
\hline Cardiac death due to bleeding & $6(0.06)$ & $2(0.07)$ & $2(0.03)$ & \\
\hline Non-cardiac death & $75(1)$ & $19(1)$ & $24(0.4)$ & 0.008 \\
\hline Death for unknown reason & $6(0.06)$ & $2(0.07)$ & $6(0.09)$ & \\
\hline All-cause death (1 year) & $752(7)$ & $214(7)$ & $243(4)$ & $<0.001$ \\
\hline Cardiac death & $523(5)$ & $128(4)$ & $115(2)$ & $<0.001$ \\
\hline Cardiac death due to ACS & $309(3)$ & $74(2.4)$ & $28(0.4)$ & \multirow{6}{*}{$<0.001$} \\
\hline Cardiac death due to sudden cardiac arrest & $35(0.3)$ & $9(0.3)$ & $20(0.3)$ & \\
\hline Cardiac death due to $\mathrm{AHF}$ & $134(1.3)$ & $40(1.3)$ & $49(0.7)$ & \\
\hline Cardiac death due to stroke & $2(0.02)$ & $1(0.03)$ & $1(0.01)$ & \\
\hline Cardiac death due to procedural complications & $3(0.03)$ & $0(0)$ & $3(0.04)$ & \\
\hline Cardiac death due to bleeding & $8(0.1)$ & $2(0.1)$ & $3(0.04)$ & \\
\hline Non-cardiac death & $263(2.6)$ & $85(2.8)$ & $133(2.0)$ & $<0.001$ \\
\hline Death for unknown reason & $46(0.4)$ & $19(0.6)$ & $35(0.5)$ & $<0.001$ \\
\hline Definite or probable stent thrombosis & $31(0.3)$ & $4(0.1)$ & $13(0.2)$ & 0.15 \\
\hline Non-fatal ACS & $133(1)$ & $53(2)$ & $131(2)$ & $<0.001$ \\
\hline STEMI & $76(1)$ & $16(1)$ & $13(0.2)$ & \multirow{3}{*}{$<0.001$} \\
\hline NSTEMI & $33(0.3)$ & $23(1)$ & $28(0.4)$ & \\
\hline UA & $41(0.4)$ & $21(1)$ & $101(1)$ & \\
\hline Non-fatal ischemic stroke & $93(1)$ & $23(1)$ & $46(1)$ & 0.25 \\
\hline Non-fatal bleeding & $171(2)$ & $43(1)$ & $122(2)$ & 0.40 \\
\hline Access site bleeding & $19(0.2)$ & $6(0.2)$ & $12(0.2)$ & \multirow{6}{*}{0.83} \\
\hline Upper GI & $25(0.2)$ & $8(0.3)$ & $20(0.3)$ & \\
\hline Lower Gl & $48(0.5)$ & $15(0.5)$ & $41(0.6)$ & \\
\hline Intracranial & $22(0.2)$ & $3(0.1)$ & $14(0.2)$ & \\
\hline Urogenital & $22(0.2)$ & $3(0.1)$ & $18(0.3)$ & \\
\hline Other & $47(0.5)$ & $10(0.3)$ & $30(0.4)$ & \\
\hline Non-fatal AHF & $238(2)$ & $78(3)$ & $119(2)$ & 0.01 \\
\hline Planned revascularization & $1,774(17)$ & $563(19)$ & $1,269(19)$ & 0.04 \\
\hline Staged PCl & $193(2)$ & $81(3)$ & $268(4)$ & \multirow{5}{*}{$<0.001$} \\
\hline $\mathrm{PCl}$ due to chest discomfort symptoms & $419(4)$ & $124(4)$ & $241(4)$ & \\
\hline $\mathrm{PCl}$ due to proven myocardial ischemia & $132(1)$ & $40(1)$ & $104(2)$ & \\
\hline $\mathrm{PCl}$ without symptoms/ischemia & $1,044(1)$ & $315(1)$ & $666(1)$ & \\
\hline CABG & $87(1)$ & $36(1)$ & $58(1)$ & \\
\hline 3-point MACE & $608(6)$ & $164(5)$ & $168(2)$ & $<0.001$ \\
\hline
\end{tabular}

Unless indicated otherwise, data are given as $\mathrm{n}(\%)$. ACS, acute coronary syndrome; AHF, acute heart failure; GI, gastrointestinal; MACE, major adverse cardiac events. Other abbreviations as in Table 1.

testinal bleeding, cerebrovascular or spinal bleeding, urogenital bleeding, and bleeding of unknown origin.

\section{Statistical Analysis}

Descriptive analysis was performed to compare baseline patient and coronary lesion characteristics, details on specific procedures used, and 1-year outcomes among the STEMI, NSTEMI, and UA groups. Variables are expressed as the mean $\pm \mathrm{SD}$, median and interquartile range (IQR), or counts and percentages, as appropriate. For comparisons among groups, the $\chi^{2}$ test was used for categorical variables and 1-way analysis of variance was used for continu- ous variables.

One-year rates of all-cause death, individual modes of death, ACS, stroke, bleeding, acute heart failure, and repeat revascularization were calculated using the KaplanMeier method. Unadjusted and adjusted Cox proportional hazard ratios (HRs) and Fine-Gray competing risks regression models were used to calculate effect estimates as cause-specific HR for Cox models or subdistribution HR for Fine-Gray models, ${ }^{18}$ with $95 \%$ confidence intervals (CI). To account for the multisite design, all models included the registration hospital site as a random intercept. Univariable and multivariable HRs were calculated, 


\begin{tabular}{|c|c|c|c|c|c|}
\hline & \multirow{2}{*}{$\begin{array}{c}\text { UA } \\
(n=6,773)\end{array}$} & \multicolumn{2}{|c|}{ NSTEMI $(n=3,027)$} & \multicolumn{2}{|c|}{ STEMI $(n=10,242)$} \\
\hline & & HR (95\% Cl) & $P$ value & HR (95\% Cl) & $P$ value \\
\hline \multicolumn{6}{|l|}{ All-cause death } \\
\hline Univariable Cox regression analysis & Reference & $1.97(1.63-2.39)$ & $<0.001$ & $2.10(1.81-2.43)$ & $<0.001$ \\
\hline Multivariable Cox regression analysis & Reference & $1.57(1.29-1.92)$ & $<0.001$ & $1.91(1.62-2.26)$ & $<0.001$ \\
\hline \multicolumn{6}{|l|}{ Cardiac death } \\
\hline Unadjusted Cox regression analysis & Reference & $2.75(2.09-3.63)$ & $<0.001$ & $3.27(2.62-4.09)$ & $<0.001$ \\
\hline Multivariable Cox regression analysis & Reference & $1.94(1.45-2.61)$ & $<0.001$ & $2.62(2.04-3.36)$ & $<0.001$ \\
\hline \multicolumn{6}{|l|}{ Non-cardiac death } \\
\hline Unadjusted Cox regression analysis & Reference & $1.48(1.14-1.93)$ & $<0.001$ & $1.31(1.07-1.61)$ & $<0.001$ \\
\hline Multivariable Cox regression analysis & Reference & $1.40(1.06-1.84)$ & $<0.001$ & $1.43(1.14-1.80)$ & $<0.001$ \\
\hline \multicolumn{6}{|c|}{ 3-point MACE: Cardiac death, non-fatal MI, stroke } \\
\hline Unadjusted Cox regression analysis & Reference & $2.17(1.73-2.71)$ & $<0.001$ & $2.37(1.99-2.83)$ & $<0.001$ \\
\hline Multivariable Cox regression analysis & Reference & $1.68(1.33-2.13)$ & $<0.001$ & $2.12(1.74-2.59)$ & $<0.001$ \\
\hline \multicolumn{6}{|l|}{ Non-fatal ACS } \\
\hline Unadjusted analysis & Reference & $0.99(0.71-1.39)$ & 0.97 & $0.64(0.49-0.84)$ & 0.001 \\
\hline Multivariable Cox regression analysis & Reference & $1.03(0.74-1.42)$ & 0.79 & $0.89(0.67-1.19)$ & 0.44 \\
\hline \multicolumn{6}{|l|}{ Non-fatal ischemic stroke } \\
\hline Unadjusted Cox regression analysis & Reference & $1.16(0.69-1.97)$ & 0.57 & $1.45(1.04-2.01)$ & 0.03 \\
\hline Multivariable Cox regression analysis & Reference & $1.11(0.65-1.89)$ & 0.71 & $1.70(1.24-2.33)$ & 0.001 \\
\hline \multicolumn{6}{|l|}{ Non-fatal AHF } \\
\hline Unadjusted Cox regression analysis & Reference & $1.42(1.04-1.92)$ & 0.03 & $1.38(1.10-1.73)$ & 0.005 \\
\hline Multivariable Cox regression analysis & Reference & $1.48(1.12-1.97)$ & 0.007 & $1.78(1.38-2.29)$ & $<0.001$ \\
\hline \multicolumn{6}{|l|}{ Non-fatal bleeding } \\
\hline Unadjusted Cox regression analysis & Reference & $0.80(0.53-1.20)$ & 0.29 & $0.99(0.76-1.29)$ & 0.94 \\
\hline Multivariable Cox regression analysis & Reference & $0.80(0.53-1.23)$ & 0.32 & $1.08(0.82-1.42)$ & 0.59 \\
\hline \multicolumn{6}{|l|}{ Repeat revascularization } \\
\hline Unadjusted Cox regression analysis & Reference & $1.05(0.92-1.20)$ & 0.49 & $0.98(0.89-1.08)$ & 0.66 \\
\hline Multivariable Cox regression analysis & Reference & $0.96(0.84-1.09)$ & 0.55 & $0.98(0.89-1.08)$ & 0.74 \\
\hline
\end{tabular}

$\mathrm{Cl}$, confidence interval; HR, hazard ratio. Other abbreviations as in Tables 1,3.

with the latter adjusting for covariates associated with inhospital mortality previously published from the J-PCI Registry, ${ }^{19}$ which included age, sex, previous PCI, coronary artery bypass graft surgery, hypertension, dyslipidemia, smoking, CKD, hemodialysis, peripheral vascular disease, previous heart failure, cardiogenic shock, 3-vessel disease, left main trunk (LMT) lesion, performance of PCI to the LMT, and PCI for a proximal left anterior descending (LAD) artery lesion.

All data were managed by the National Clinical Database and by data analysts belonging to the J-PCI Registry statistical analysis team. All analyses were performed using Stata/IC Version 15.1 for Macintosh (StataCorp, College Station, TX, USA). $\mathrm{P}$ values are 2 -sided and $\mathrm{P}<0.05$ was considered statistically significant.

\section{Results}

\section{Baseline Patient Characteristics}

In the J-PCI OUTCOME cohort, 49,014 patients were followed up, comprising 16,129 thousand-person days (follow-up rate $79.3 \%$; Figure 1). Within the 20,042 ACS patients $(51 \%$ [n=10,242] STEMI, $15 \%[\mathrm{n}=3,027]$ NSTEMI, and $34 \%[n=6,773]$ UA), the mean age was $69.6 \pm 12.4$ years, $23 \%$ were aged $>80$ years, $76 \%$ were male, $20 \%$ had a previous history of PCI, 39\% had diabetes, $18 \%$ had CKD, and $4 \%$ were on hemodialysis. Cardiogenic shock and cardiac arrest occurred in $8 \%$ and $5 \%$ of study patients, respectively. Antiplatelet medications were administered within $24 \mathrm{~h}$ of PCI for $81 \%$ of STEMI patients, $86 \%$ of NSTE-ACS patients, and $90 \%$ of UA patients (Table 1). Aspirin and prasugrel were the most preferred antiplatelet agents over clopidogrel and ticagrelor for all 3 indications. The transradial approach and drug-eluting stents (DES) were used in $65 \%$ and $86 \%$ of all cases, respectively (Table 2 ).

STEMI patients were younger, predominantly male, with a smaller proportion of previous coronary artery disease and comorbidities, and reported a higher proportion of cardiogenic shock and cardiac arrest events than patients in the other 2 groups. The proportion of PCI procedures performed via the transfemoral approach and the use of thrombus aspiration and distal protection devices was highest among STEMI patients, although the implantation rate of DES did not differ significantly among the groups. NSTEMI patients were older (mean age $70.0 \pm 12.5$ years), with $26 \%$ aged above 80 years, and received the greatest proportion of multivessel PCI. Finally, the UA patient group had the highest proportion of cases with previous PCI, diabetes, hemodialysis, and the presence of extracardiac vascular lesions, as well as highest rate of administration of antiplatelet therapy prior to PCI.

\section{Post-PCI 30-Day and 1-Year Mortality Rates}

The overall 30-day all-cause, cardiac, and non-cardiac mortality rates were $3.0 \%, 2.4 \%$, and $0.6 \%$, respectively. Fatal ACS, fatal acute heart failure, and sudden cardiac 
A All-cause death

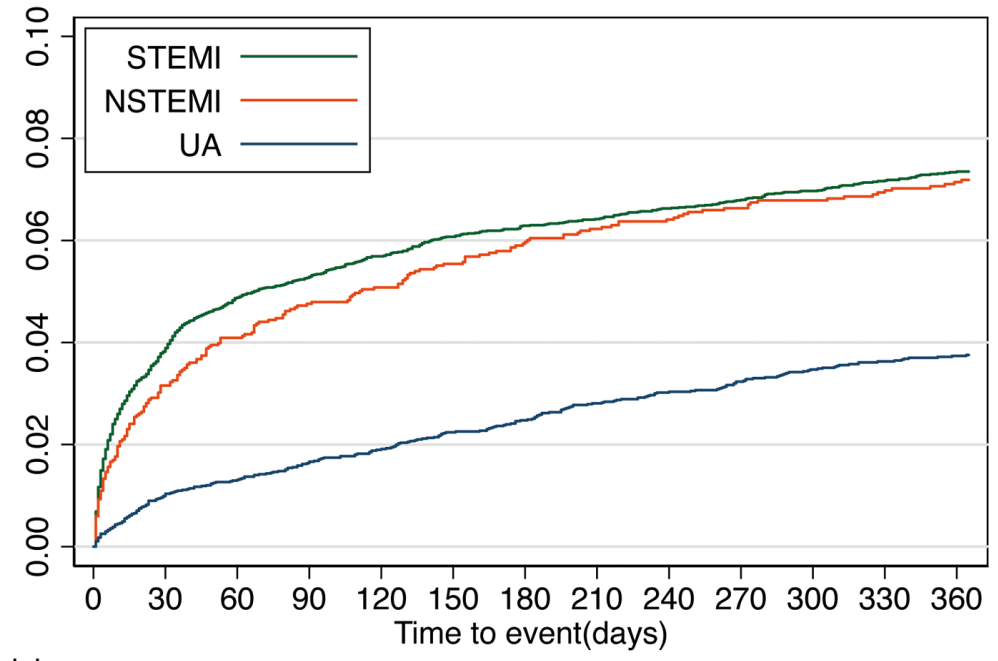

Number at risk

STEMI10185

NSTEMI 3015

UA 6762

\section{6}

2701

6369

B. Cardiac death

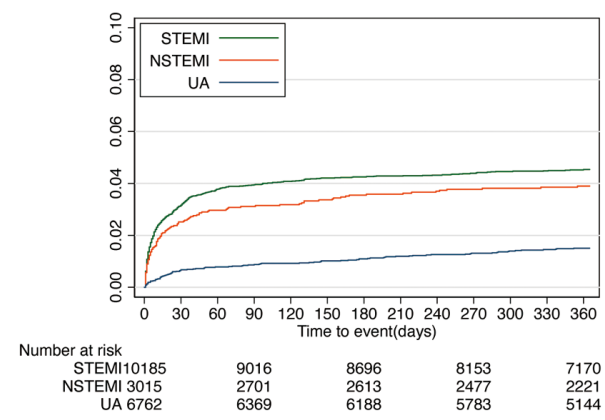

C. Non-cardiac death

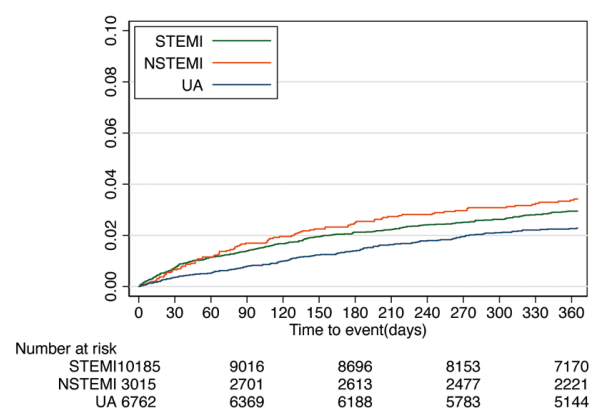

Figure 2. Kaplan-Meier curves for (A) all-cause, (B) cardiac, and (C) non-cardiac deaths according to indication. NSTEMI, nonST segment elevation myocardial infarction; STEMI, ST-elevation myocardial infarction; UA, unstable angina.

events were the leading modes of cardiac death. Cardiac deaths predominated over non-cardiac deaths $(81 \%$ vs. $19 \%$ ) among all deaths within the first month after PCI. STEMI patients had the highest 30-day all-cause, cardiac, and non-cardiac mortality rates $(4.2 \%, 3.5 \%$, and $0.7 \%$, respectively). NSTEMI patients had the second highest rates, and UA patients had the lowest mortality rates (Table 3).

The overall 1-year incidence of all-cause, cardiac, and non-cardiac mortality rates was $7.1 \%, 4.2 \%$, and $2.8 \%$, respectively (Table 3). An acute increase in the incidence of cardiac deaths was observed within the first 30 days, followed by a plateau with a minimal increase for all indications. In contrast with cardiac deaths, the incidence of non-cardiac deaths exhibited a gradual and constant increase across all indications. The risk-adjusted HRs for 1-year all-cause death, cardiac death, and non-cardiac death were all significantly higher in STEMI and NSTEMI patients than in UA patients (Table 4; Figure 2). The adjusted HRs for cardiac death were particularly higher for STEMI (HR 2.62; 95\% CI 2.04-3.36) and NSTEMI (HR 1.94; 95\% CI 1.45-2.61) patients than for UA patients.

\section{Clinical Events at 1 Year}

Kaplan-Meier and cumulative incidence curves of nonfatal adverse events, including ACS, ischemic stroke, acute heart failure, major bleeding, and repeat revascularization, are shown in Figures 3,4. The unadjusted and adjusted HRs for subsequent 1-year adverse events accounting for confounders and registered hospitals as random intercepts are presented in Table 4. Of note, compared with UA patients, STEMI patients had higher risks for all fatal events, non-fatal ischemic stroke, and acute heart failure, 
A Acute coronary syndrome

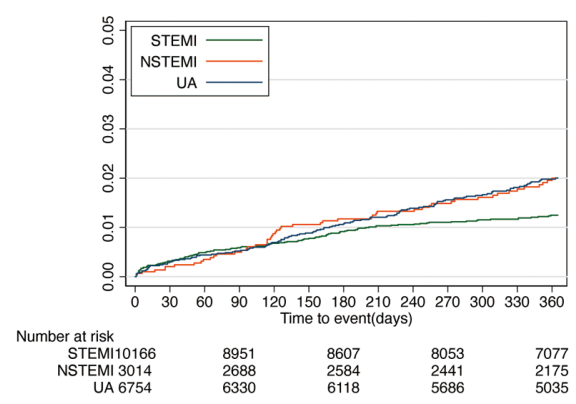

C Acute heart failure

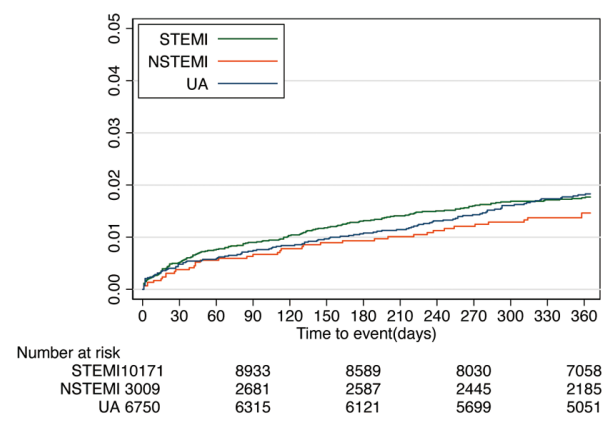

E Repeat revascularization

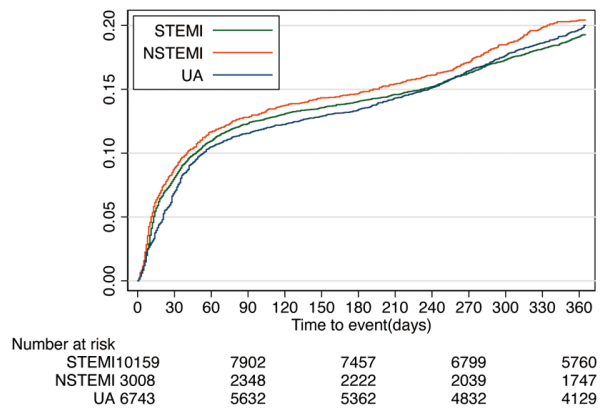

B Stroke

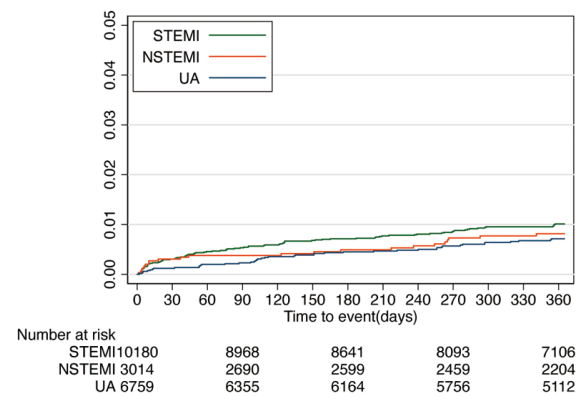

D Bleeding

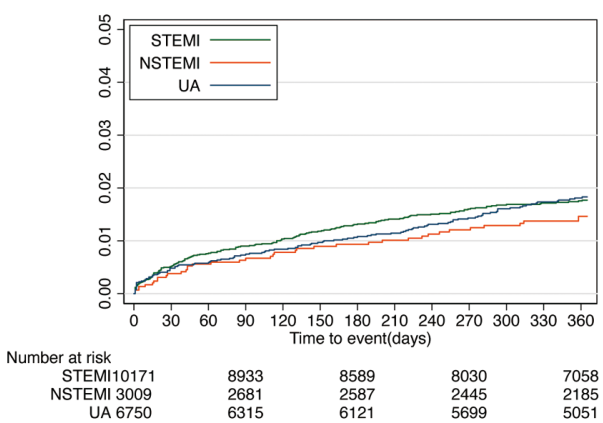

Figure 3. Kaplan-Meier curves of non-fatal events: (A) acute coronary syndrome, (B) stroke, (C) acute heart failure, (D) bleeding, and $(\mathbf{E})$ repeat vascularization. NSTEMI, non-ST segment elevation myocardial infarction; STEMI, ST-elevation myocardial infarction; UA, unstable angina.

and NSTEMI patients had a higher risk for heart failure. Notably, a history of previous heart failure, CKD, and cardiogenic shock at hospital presentation were associated with a higher risk of 1-year major adverse cardiac events (MACE) in all 3 subcategories of ACS (Supplementary Table 2). Specifically, for STEMI patients, older age, the presence of 3-vessel disease, and performing PCI for either the LMT or LAD \#6 lesion were associated with higher risks of 1-year MACE. For NSTEMI patients, PCI for an LAD \#6 lesion was associated with higher risks. Finally, for UA patients, older age, female sex, and a history of previous PCI were associated with higher risks of 1-year MACE.

Definite and probable stent thrombosis occurred in $0.3 \%, 0.1 \%$, and $0.2 \%$ of STEMI, NSTEMI, and UA patients, respectively, within the 1-year of follow-up. Most cases of stent thrombosis occurred in the first 30 days, although there were a number of cases in the later months seen as late stent thrombosis. With regard to repeat revascularization, $18 \%$ of all ACS patients underwent repeat 
A Acute coronary syndrome

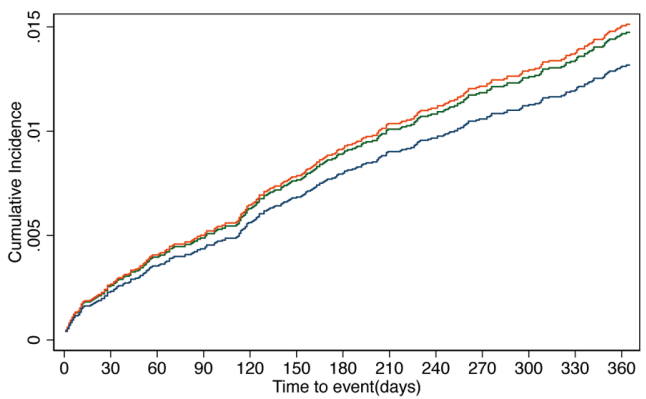

C Acute heart failure

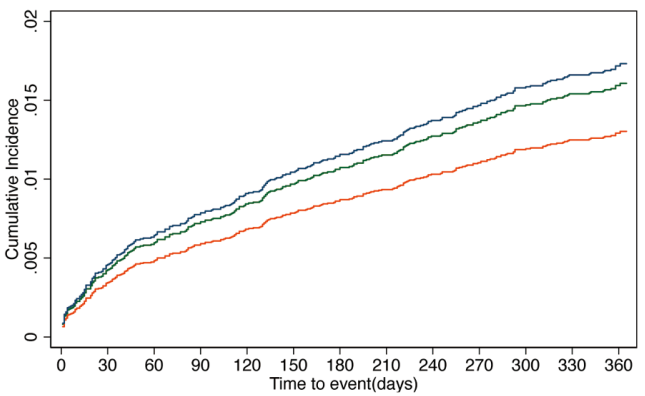

E Repeat revascularization

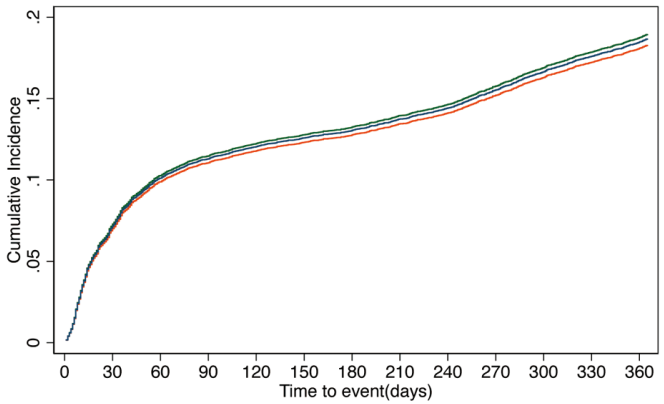

B Stroke

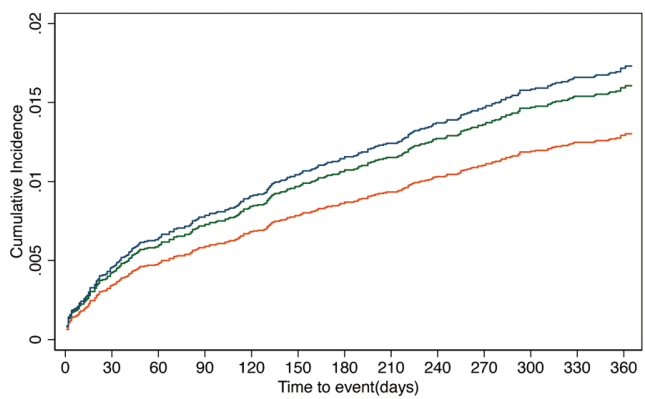

D Bleeding

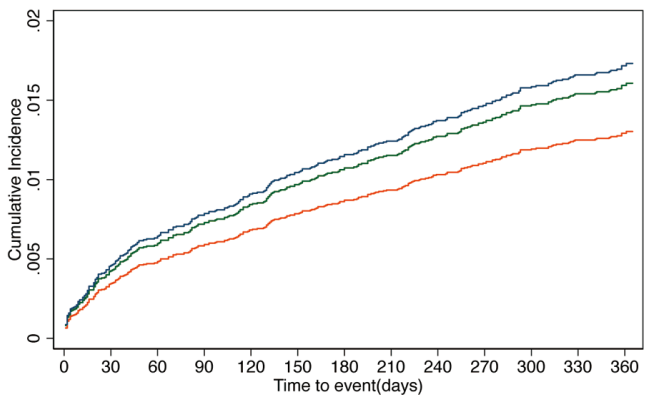

Figure 4. Cumulative incidence of non-fatal events: (A) acute coronary syndrome, (B) stroke, (C) acute heart failure, (D) bleeding, and $(\mathbf{E})$ repeat vascularization. NSTEMI, non-ST segment elevation myocardial infarction; STEMI, ST-elevation myocardial infarction; UA, unstable angina.

revascularization within 1 year of the index PCI. Staged PCI was performed in approximately $10 \%$ of patients within 30 days, followed by a proportional increase in repeat PCI due to chest discomfort and proven myocardial ischemia in the past 6 months.

\section{Discussion}

The improvement in early in-hospital outcomes of ACS patients has been largely attributed to the widespread distribution of PCI-capable hospitals and their expertise in performing prompt revascularization. Nonetheless, data on late outcomes in the real-world setting have remained scarce in the modern era. This study is the first to evaluate a nationwide cohort extending beyond the limitations of previous representative regional registries, such as the Coronary Revascularization Demonstrating Outcome StudyKyoto Registry Cohort (CREDO-KYOTO)-1, -2, and -3 and the Japan Cardiovascular Database Keio interhospital Cardiology Study (JCD-KiCS), and collected 1-year outcomes from consecutive PCI patients during 2017 covering all 47 prefectures from 8 regions of Japan. We report that for ACS hospital survivors, cardiac deaths were the main cause of 30-day mortality with ACS, whereas heart failure and sudden cardiac death were the leading modes of cardiac death, particularly among STEMI and NSTEMI 
patients. The risk of 1-year subsequent adverse events was generally higher in STEMI and NSTEMI patients than in UA patients.

The crude 30-day and 1-year all-cause mortality and thrombotic event rates, including incident ACS, in the present study were comparable to or lower than those in previous reports from representative real-world registries. The 30-day mortality rate among acute myocardial infarction (AMI) patients was reported to be $7.1 \%$ in the Osaka Acute Coronary Insufficiency Study ${ }^{20}$ (patients registered from January 2002 to December 2010 from 25 hospitals) and $9.4 \%$ in the HIJAMI Study ${ }^{21}$ (patients registered from January 1999 to June 2001 from 17 hospitals). Although direct comparison of long-term outcomes between databases is challenging due to differences in patient registration methods, race, ethnicity, timing of registration, approved medications or treatment device, and level of access to care, these numbers are approximately half or one-third the rates reported in studies conducted over a decade ago, with the exception of the Prevention of AtherothrombotiC Incidents Following Ischemic Coronary attack registry (2008-2009 from 98 hospitals). ${ }^{22}$ The results of the present study are also comparable to those of a report from the SWEDEHEART registry (2008), which registered all AMI patients aged 60-69 years, ${ }^{23}$ and the NCDR CathPCI Registry Medicare beneficiaries (20092014). ${ }^{24}$

Cardiac deaths occurred frequently during the first 30 days and plateaued thereafter. In contrast, non-cardiac deaths showed an unchanged constant upward trend that overlapped the incidence of cardiac deaths at 1 year after the index PCI. A similar observation has been reported in a single-center study at the Mayo Clinic, ${ }^{25}$ as well as in a pooled randomized trial database analysis from the Cardiovascular Research Foundation, which demonstrated that the predominant cause of death switched 3 years after PCI. ${ }^{26}$ The results of the present study re-emphasize the importance of the periodic assessment of long-term causes of specific causes of death and adverse events, because the proportion of these events changes over time. It also underscores the need for the adjustment of treatment thresholds for specific interventions to obtain maximal effect at a given time period.

The leading attributable causes of cardiac deaths were death due to recurrent ACS, heart failure, and sudden cardiac death. In line with the TIMI Study Group NSTEACS merged patient database, recurrent ACS was the leading cause of 30-day cardiac mortality in the present study. At 30 days after the index procedure, the proportion of leading modes of cardiac death shifted towards heart failure and sudden cardiac deaths. Sudden cardiac deaths occur in the early stage after myocardial infarction, and were observed in our database. Nonetheless, intracardiac defibrillator implantation for primary prevention purposes $<40$ days after myocardial infarction or $<90$ days after PCI is prohibited in the $\mathrm{US}^{27}$ based on the results of the 2004 Defibrillator in Acute Myocardial Infarction Trial (DINAMIT) $^{28}$ trial and the 2009 Immediate Risk-Stratification Improves Survival (IRIS) trial. ${ }^{29}$ This unmet need may be mitigated through several of the JCS guidelines, ${ }^{30}$ which propose secondary prevention strategies such as: (1) adherence and uptitration of optimal medical therapies (OMTs) including aspirin, $\mathrm{P}_{2} \mathrm{Y}_{12}$ inhibitors, statins, and renin-angiotensin-aldosterone system inhibitors (RAASi);31 (2) participation in cardiac rehabilitation programs; ${ }^{\mathbf{3 2}}$ and
(3) application of wearable cardioverter-defibrillators, if tolerable, in select patients. The 2018 Vest Prevention of Early Sudden Death Trial (VEST) did not show any significant beneficial effects of wearable cardioverter-defibrillators in post-myocardial infarction patients, with an approximate $90 \%$ prescription rate of statins, $\beta$-blockers, and RAASi. ${ }^{33}$ That study suggested that medical therapy is indeed a key component for reducing sudden cardiac deaths and for those who can tolerate the devices.

The timing and incidence of stent thrombosis were similar to those observed in the case-control Retrospective Multicenter Registry of ST After First- and Second-Generation DES Implantation (REAL-ST) study, including 313 definite stent thromboses during a mix of the first- and second-generation DES eras. ${ }^{34}$ Thoughtful consideration of balancing individual bleeding and thrombotic risks is needed when considering the continuation or discontinuation of dual antiplatelet agents after $\mathrm{PCI}^{30}$ by using locally validated risk scores, such as the PRECISE-DAPT score, ${ }^{35}$ to minimize overall long-term treatment-related complications. Despite treatment improvement with P2Y 12 inhibitors $^{36}$ and newer generation drug-eluting coronary stents, ${ }^{37}$ the identification of high-risk patients predisposed to ACS recurrence, including stent thrombosis, remains a challenge.

\section{Study Limitations}

The findings of this study should be interpreted in the context of several limitations. First, although study patients and hospitals were chosen from diverse backgrounds, there may have been selection bias. The hospitals included in the J-PCI OUTCOME registry were generally moderate- to high-volume centers that chose to take part in this unique nationwide registry. The nature of such site selection may have introduced bias towards better outcomes compared with other hospitals not included in the study. Nonetheless, differences in patient characteristics observed between this cohort and the 2017 cross-sectional registry were all within expectation, with the former have a higher proportion of ACS and the latter have a higher proportion of elective cases.

Second, outcome events were not extensively adjudicated by a central adjudicating committee; however, randomly selected site visits are performed every year to a total of approximately 20 hospitals to ensure data completeness and integrity. Validation of data reliability remains a future issue; nonetheless, this is the most recent and the largest nationwide cohort study conducted to evaluate extensive 1-year outcomes in patients at moderate- to high-volume PCI hospitals.

Third, the performance of complete revascularization for ACS patients that received Class IIa recommendation from the CVIT expert consensus document is unknown. Furthermore, the effect of repeat revascularization, including staged PCI for residual lesions or de novo coronary stenosis lesions, was not evaluated. Fourth, details on left ventricle ejection fraction, cardiac enzymes, discharge medications, and uptitration of OMT likely to affect longterm prognosis are unknown. This may be one of the most actionable issues to improve the quality of care, because previous reports have demonstrated undertreatment, particularly in individuals who are older and present renal dysfunction. ${ }^{38}$

Fifth, the duration of dual antiplatelet therapy is unknown within our database and is likely affected by long-term thromboembolic and major bleeding events. 
Sixth, bleeding events were not defined according to the standardized bleeding criteria, such as the Bleeding Academic Research Consortium, Global Use of Strategies to Open Occluded Arteries, or Thrombolysis in Myocardial Infarction, due to a lack of feasibility in a nationwide cohort. Finally, our ACS population and 1-year outcomes should be interpreted in the context of patients in whom PCI was deemed feasible at the time of presentation, with patients who underwent emergency coronary artery bypass grafting surgery or conservative medical therapy only not included.

\section{Conclusions}

In this nationwide cohort, cardiac deaths were the main cause of 30-day mortality, with ACS, heart failure, and sudden cardiac death as the leading causes of cardiac deaths among ACS hospital survivors. The risk of 1-year subsequent adverse events was generally higher in STEMI and NSTEMI patients than in UA patients. Future research is warranted to improve the quality of secondary prevention for the AMI population in the modern era.

\section{Acknowledgments}

The authors thank all the participating hospitals for their efforts in creating the J-PCI OUTCOME cohort. A full list of all hospitals is available as Supplementary Appendix.

\section{Sources of Funding}

This work was supported by the Japanese Association of Cardiovascular Intervention and Therapeutics, the Japan Agency for Medical Research and Developments (Grant no. 17ek0210097 h0001), and by the JSPS KAKENHI Grant Number JP18K17332. There are no relationships with industry.

\section{Disclosures}

M.S. received a Japan Promotional Society for Cardiovascular Disease Sakakibara Memorial Research Grant, a grant from the Japan Heart Foundation, and a JSPS KAKENHI Grant Number JP18K17332 during the conduct of the study, as well as grants from Takeda Pharma and Takeda Japan Medical Office Funded Research Grant 2018 not relevant to this study. S.K. has received investigator-initiated grant funding from Bayer and Daiichi Sankyo and personal consulting fees from Bayer and Bristol-Myers Squibb. H.I. has received lecture fees from Astellas Pharma, AstraZeneca, Bayer, Bristol-Myers Squibb, Chugai Pharma, Daiichi Sankyo, Otsuka Pharma, Pfizer, Mochida Pharma, MSD, and JSPS KAKENHI Grant Number JP21K08064. T.A. has received lecture fees from Astellas Pharma, AstraZeneca, Bayer, Daiichi Sankyo, and Bristol-Myers Squibb. T.I. has received a research grant from Boston Scientific. M.N. has received remuneration for lectures from Daiichi Sankyo, Sanofi, Bayer, Nippon Boehringer Ingelheim, Bristol-Myers Squibb, Terumo, Japan Lifeline, Abbott, Boston Scientific, Medtronic, and Nipro, and investigator-initiated grant funding from Sanofi and Daiichi Sankyo. H.I., T.A., and Y.I. are members of Circulation Journal's Editorial Team. The remaining authors have no conflicts of interest to report.

\section{IRB Information}

The study protocol was approved by a third-party central ethics committee at the Clinical Research Promotion Network Japan (Reference no. CVIT-2017) and local institutional review boards of each participating site. The names of the individual sites are provided in the Supplementary Appendix. Patient consent was obtained through an opt-out format notification on websites and posters, and procedures were conducted according to the principle of the Declaration of Helsinki.

\section{Data Availability}

The deidentified participant data will not be shared.

\section{References}

1. The Lancet. 40 years of percutaneous coronary intervention:
Where next? Lancet 2017; 390: 715.

2. Benjamin EJ, Virani SS, Callaway $\mathrm{CW}$, Chamberlain AM, Chang AR, Cheng S, et al. Heart disease and stroke statistics-2018 update: A report from the American Heart Association. Circulation 2018; 137: e67-e492.

3. Ozaki Y, Katagiri Y, Onuma Y, Amano T, Muramatsu T, Kozuma K, et al. CVIT expert consensus document on primary percutaneous coronary intervention (PCI) for acute myocardial infarction (AMI) in 2018. Cardiovasc Interv Ther 2018; 33: 178-203.

4. Berg DD, Wiviott SD, Braunwald E, Guo J, Im K, Kashani A, et al. Modes and timing of death in 66252 patients with non-STsegment elevation acute coronary syndromes enrolled in 14 TIMI trials. Eur Heart J 2018; 39: 3810-3820.

5. Pilgrim T, Vranckx P, Valgimigli M, Stefanini GG, Piccolo R, Rat J, et al. Risk and timing of recurrent ischemic events among patients with stable ischemic heart disease, non-ST-segment elevation acute coronary syndrome, and ST-segment elevation myocardial infarction. Am Heart J 2016; 175: 56-65.

6. Alkhouli M, Alqahtani F, Kalra A, Gafoor S, Alhaji M, Alreshidan $\mathrm{M}$, et al. Trends in characteristics and outcomes of patients undergoing coronary revascularization in the United States, 2003-2016. JAMA Netw Open 2020; 3: e1921326.

7. Fokkema ML, James SK, Albertsson P, Akerblom A, Calais F, Eriksson $\mathrm{P}$, et al. Population trends in percutaneous coronary intervention: 20-year results from the SCAAR (Swedish Coronary Angiography and Angioplasty Registry). J Am Coll Cardiol 2013; 61: $1222-1230$.

8. Hira RS, Bhatt DL, Fonarow GC, Heidenreich PA, Ju C, Virani SS, et al. Temporal trends in care and outcomes of patients receiving fibrinolytic therapy compared to primary percutaneous coronary intervention: Insights from the Get With The Guidelines Coronary Artery Disease (GWTG-CAD) Registry. $J$ Am Heart Assoc 2016; 5: 004113

9. Inoue T, Kuwabara H, Fushimi K. Regional variation in the use of percutaneous coronary intervention in Japan. Circ J 2017; 81: 195-198.

10. Sawano M, Yamaji K, Kohsaka S, Inohara T, Numasawa Y, Ando $\mathrm{H}$, et al. Contemporary use and trends in percutaneous coronary intervention in Japan: An outline of the J-PCI registry. Cardiovasc Interv Ther 2020; 35: 218-226.

11. Yamaji K, Kohsaka S, Morimoto T, Fujii K, Amano T, Uemura $\mathrm{S}$, et al. Relation of ST-segment elevation myocardial infarction to daily ambient temperature and air pollutant levels in a Japanese Nationwide Percutaneous Coronary Intervention Registry. Am J Cardiol 2017; 119: 872-880.

12. Inohara T, Kohsaka S, Yamaji K, Amano T, Fujii K, Oda H, et al. Impact of institutional and operator volume on short-term outcomes of percutaneous coronary intervention: A report from the Japanese Nationwide Registry. JACC Cardiovasc Interv 2017; 10: 918-927.

13. Numasawa $\mathrm{Y}$, Inohara $\mathrm{T}$, Ishii $\mathrm{H}$, Yamaji $\mathrm{K}$, Kohsaka $\mathrm{S}$, Sawano M, et al. Comparison of outcomes after percutaneous coronary intervention in elderly patients, including 10628 nonagenarians: Insights from a Japanese Nationwide Registry (J-PCI Registry). J Am Heart Assoc 2019; 8: e011183.

14. Murakami A, Hirata Y, Motomura N, Miyata H, Iwanaka T, Takamoto S. The National Clinical Database as an initiative for quality improvement in Japan. Korean J Thorac Cardiovasc Surg 2014; 47: 437-443.

15. Inohara T, Kohsaka S, Yamaji K, Amano T, Fujii K, Oda H, et al. Impact of institutional and operator volume on short-term outcomes of percutaneous coronary intervention: A report from the Japanese Nationwide Registry. JACC Cardiovasc Interv 2017; 10: 918-927.

16. Hicks KA, Mahaffey KW, Mehran R, Nissen SE, Wiviott SD, Dunn B, et al. 2017 Cardiovascular and stroke endpoint definitions for clinical trials. Circulation 2018; 137: 961-972.

17. Garcia-Garcia HM, McFadden EP, Farb A, Mehran R, Stone $\mathrm{GW}$, Spertus J, et al. Standardized end point definitions for coronary intervention trials: The Academic Research Consortium-2 Consensus Document. Circulation 2018; 137: 2635-2650.

18. Robins JM, Hernan MA, Brumback B. Marginal structural models and causal inference in epidemiology. Epidemiology 2000; 11: $550-560$.

19. Inohara T, Kohsaka S, Yamaji K, Ishii H, Amano T, Uemura S, et al. Risk stratification model for in-hospital death in patients undergoing percutaneous coronary intervention: A nationwide retrospective cohort study in Japan. BMJ Open 2019; 9: e026683.

20. Kinjo K, Sato H, Sato H, Ohnishi Y, Hishida E, Nakatani D, et al. Prognostic significance of atrial fibrillation/atrial flutter in 
patients with acute myocardial infarction treated with percutaneous coronary intervention. Am J Cardiol 2003; 92: 1150-1154.

21. Kasanuki H, Honda T, Haze K, Sumiyoshi T, Horie T, Yagi M, et al. A large-scale prospective cohort study on the current status of therapeutic modalities for acute myocardial infarction in Japan: Rationale and initial results of the HIJAMI Registry. Am Heart J 2005; 150: 411-418.

22. Daida H, Miyauchi K, Ogawa H, Yokoi H, Matsumoto M, Kitakaze M, et al. Management and two-year long-term clinical outcome of acute coronary syndrome in Japan: Prevention of atherothrombotic incidents following ischemic coronary attack (PACIFIC) registry. Circ J 2013; 77: 934-943.

23. Jernberg $\mathrm{T}$, Attebring MF, Hambraeus $\mathrm{K}$, Ivert $\mathrm{T}$, James $\mathrm{S}$, Jeppsson A, et al. The Swedish Web-system for Enhancement and Development of Evidence-based care in Heart disease Evaluated According to Recommended Therapies (SWEDEHEART). Heart 2010; 96: 1617-1621.

24. Fanaroff AC, Zakroysky P, Wojdyla D, Kaltenbach LA, Sherwood MW, Roe MT, et al. Relationship between operator volume and long-term outcomes after percutaneous coronary intervention. Circulation 2019; 139: 458-472.

25. Spoon DB, Psaltis PJ, Singh M, Holmes DRJ, Gersh BJ, Rihal CS, et al. Trends in cause of death after percutaneous coronary intervention. Circulation 2014; 129: 1286-1294.

26. Brener SJ, Tarantini G, Leon MB, Serruys PW, Smits PC, von Birgelen C, et al. Cardiovascular and noncardiovascular death after percutaneous coronary intervention: Insights from 32882 patients enrolled in 21 randomized trials. Circ Cardiovasc Interv 2018; 11: e006488.

27. Russo AM, Stainback RF, Bailey SR, Epstein AE, Heidenreich PA, Jessup M, et al. ACCF/HRS/AHA/ASE/HFSA/SCAI/ SCCT/SCMR 2013 appropriate use criteria for implantable cardioverter-defibrillators and cardiac resynchronization therapy: A report of the American College of Cardiology Foundation appropriate use criteria task force, Heart Rhythm Society, American Heart Association, American Society of Echocardiography, Heart Failure Society of America, Society for Cardiovascular Angiography and Interventions, Society of Cardiovascular Computed Tomography, and Society for Cardiovascular Magnetic Resonance. J Am Coll Cardiol 2013; 61: 1318-1368.

28. Hohnloser SH, Kuck KH, Dorian P, Roberts RS, Hampton JR, Hatala R, et al. Prophylactic use of an implantable cardioverterdefibrillator after acute myocardial infarction. $N$ Engl $J$ Med 2004; 351: $2481-2488$.

29. Steinbeck G, Andresen D, Seidl K, Brachmann J, Hoffmann E, Wojciechowski D, et al. Defibrillator implantation early after myocardial infarction. $N$ Engl J Med 2009; 361: 1427-1436.

30. Kimura K, Kimura T, Ishihara M, Nakagawa Y, Nakao K, Miyauchi K, et al. JCS 2018 guideline on diagnosis and treatment of acute coronary syndrome. Circ J 2019; 83: 1085-1196.

31. Yusuf S, Hawken S, Ounpuu S, Dans T, Avezum A, Lanas F, et al. Effect of potentially modifiable risk factors associated with myocardial infarction in 52 countries (the INTERHEART study): Case-control study. Lancet 2004; 364: 937-952.

32. Pouche M, Ruidavets JB, Ferrières J, Iliou MC, Douard H, Lorgis L, et al. Cardiac rehabilitation and 5-year mortality after acute coronary syndromes: The 2005 French FAST-MI study. Arch Cardiovasc Dis 2016; 109: 178-187.

33. Olgin JE, Pletcher MJ, Vittinghoff E, Wranicz J, Malik R, Morin $\mathrm{DP}$, et al. Wearable cardioverter-defibrillator after myocardial infarction. N Engl J Med 2018; 379: 1205-1215.

34. Kuramitsu S, Ohya M, Shinozaki T, Otake H, Horie K, Kawamoto $\mathrm{H}$, et al. Risk factors and long-term clinical outcomes of secondgeneration drug-eluting stent thrombosis. Circ Cardiovasc Interv 2019; 12: e007822.

35. Costa F, van Klaveren D, James S, Heg D, Raber L, Feres F, et al. Derivation and validation of the predicting bleeding complications in patients undergoing stent implantation and subsequent dual antiplatelet therapy (PRECISE-DAPT) score: A pooled analysis of individual-patient datasets from clinical trials. Lancet 2017; 389: 1025-1034.

36. Rafique AM, Nayyar P, Wang TY, Mehran R, Baber U, Berger $\mathrm{PB}$, et al. Optimal $\mathrm{P}_{2} \mathrm{Y}_{12}$ inhibitor in patients with ST-segment elevation myocardial infarction undergoing primary percutaneous coronary intervention: A network meta-analysis. JACC Cardiovasc Interv 2016; 9: 1036-1046.

37. Kandzari DE, Mauri L, Koolen JJ, Massaro JM, Doros G, Garcia-Garcia HM, et al. Ultrathin, bioresorbable polymer sirolimus-eluting stents versus thin, durable polymer everolimuseluting stents in patients undergoing coronary revascularisation (BIOFLOW V): A randomised trial. Lancet 2017; 390: 18431852.

38. Sawano M, Kohsaka S, Abe T, Inohara T, Maekawa Y, Ueda I, et al. Patterns of statin non-prescription in patients with established coronary artery disease: A report from a contemporary multicenter Japanese PCI registry. PLoS One 2017; 12: e0182687.

\section{Supplementary Files}

Please find supplementary file(s); http://dx.doi.org/10.1253/circj.CJ-21-0098 